\title{
mTORC1 serves ER stress-triggered apoptosis via selective activation of the IRE1-JNK pathway
}

\author{
H Kato ${ }^{1}$, S Nakajima ${ }^{1}$, Y Saito ${ }^{1}$, S Takahashi ${ }^{1}$, R Katoh ${ }^{2}$ and M Kitamura ${ }^{*, 1}$
}

Mammalian target of rapamycin (mTOR) has a key role in the regulation of an array of cellular function. We found that rapamycin, an inhibitor of mTOR complex 1 (mTORC1), attenuated endoplasmic reticulum (ER) stress-induced apoptosis. Among three major branches of the unfolded protein response, rapamycin selectively suppressed the IRE1-JNK signaling without affecting PERK and ATF6 pathways. ER stress rapidly induced activation of mTORC1, which was responsible for induction of the IRE1JNK pathway and apoptosis. Activation of mTORC1 reduced Akt phosphorylation, which was an event upstream of IRE-JNK signaling and consequent apoptosis. In vivo, administration with rapamycin significantly suppressed renal tubular injury and apoptosis in tunicamycin-treated mice. It was associated with enhanced phosphorylation of Akt and suppression of JNK activity in the kidney. These results disclosed that, under ER stress conditions, mTORC1 causes apoptosis through suppression of Akt and consequent induction of the IRE1-JNK pathway.

Cell Death and Differentiation (2012) 19, 310-320; doi:10.1038/cdd.2011.98; published online 22 July 2011

The endoplasmic reticulum (ER) serves several important cell functions, including protein folding, maturation and trafficking, as well as cholesterol synthesis. The quality control of newly synthesized proteins by the ER is essential for normal cell function and survival. However, integrity of the ER is often perturbed by accumulation of unfolded or misfolded proteins, leading to the unfolded protein response (UPR). ${ }^{1}$ The UPR alleviates ER stress by reducing immature proteins in the ER, whereas severe or prolonged ER stress leads to activation of the pro-apoptotic UPR and consequent cellular death. ${ }^{2}$

The UPR comprises three major signaling pathways mediated by ER type I transmembrane kinases, including inositol-requiring enzyme 1 (IRE1) and RNA-dependent protein kinase-like ER kinase (PERK), and a type II transmembrane protein, that is, activating transcription factor 6 (ATF6). ${ }^{1,3}$ IRE1 has an endoribonuclease domain and a serine/threonine kinase domain. The endoribonuclease domain removes a 26-nucleotide intron from the mRNA of $\mathrm{X}$-box binding protein 1 (XBP1), resulting in production of the spliced form of XBP1 and consequent induction of ER chaperones that reinforce ER folding capacity. The kinase domain of IRE1 activates c-Jun N-terminal kinase (JNK) via interaction with TNF receptor-associated factor 2 (TRAF2) and apoptosis signal-regulating kinase 1 (ASK1). This molecular event contributes to the induction of apoptosis.
Activation of PERK leads to phosphorylation of eukaryotic translation initiation factor $2 \alpha$ (elF2 $\alpha$ ) and causes inhibition of translation. In addition, the PERK-elF2 $\alpha$ pathway selectively induces a transcription factor ATF4 and thereby enhances expression of pro-apoptotic CCAAT/enhancer-binding protein-homologous protein (CHOP). Upon ER stress, ATF6 is transported to the Golgi apparatus where it is cleaved by site- 1 protease and site-2 protease. The cleaved cytosolic domain of ATF6 translocates into the nucleus and activates transcription of ER chaperones such as $78 \mathrm{kDa}$ glucose-regulated protein (GRP78) and GRP94. ${ }^{1,3}$

Mammalian target of rapamycin (mTOR) has a central role in the regulation of cellular processes including proliferation, differentiation and metabolism. ${ }^{4}$ It forms distinct multi-protein complexes, mTOR complex 1 (mTORC1) and mTORC2. The mTORC1 is composed of mTOR, regulatory associated protein of mTOR (Raptor) and mLST8, and is sensitive to rapamycin. mTORC 1 is one of key regulators for cell growth and metabolism thorough mTORC1-mediated direct phosphorylation of ribosomal p70S6 kinase (p70S6K) and elF4E-binding protein $1 .{ }^{5}$ The mTORC2 is composed of mTOR, Rictor, Sin1 and mLST8. It controls actin cytoskeleton organization and cell spreading, which is resistant to inhibition by rapamycin. ${ }^{5}$

Rapamycin is known as a macrolide antibiotic with potent immunosuppressive activities and has been used to prevent

\footnotetext{
${ }^{1}$ Department of Molecular Signaling, Interdisciplinary Graduate School of Medicine and Engineering, University of Yamanashi, Chuo, Yamanashi 409-3898, Japan and ${ }^{2}$ Department of Pathology, Interdisciplinary Graduate School of Medicine and Engineering, University of Yamanashi, Chuo, Yamanashi 409-3898, Japan

${ }^{*}$ Corresponding author: M Kitamura, Department of Molecular Signaling, Interdisciplinary Graduate School of Medicine and Engineering, University of Yamanashi, Shimokato 1110, Chuo, Yamanashi 409-3898, Japan. Tel/Fax: + 8155273 8054; E-mail: masanori@yamanashi.ac.jp

Keywords: Akt; endoplasmic reticulum stress; IRE1; mTOR; rapamycin

Abbreviations: ER, endoplasmic reticulum; UPR, unfolded protein response; IRE1, inositol-requiring enzyme 1; PERK, RNA-dependent protein kinase-like ER kinase; ATF6, activating transcription factor 6; XBP1, X-box binding protein 1; JNK, c-Jun N-terminal kinase; TRAF2, TNF receptor-associated factor 2; ASK1, apoptosis signalregulating kinase 1; elF2 $\alpha$, eukaryotic translation initiation factor $2 \alpha$; CHOP, CCAAT/enhancer-binding protein-homologous protein; GRP78, 78 kDa glucose-regulated protein; mTOR, mammalian target of rapamycin; mTORC1, mTOR complex 1; p70S6K, ribosomal p70S6 kinase; 4E-BP1, elF4E-binding protein 1; TSC2, tuberous sclerosis complex 2; UPRE, UPR element; Akt-CA, constitutively active Akt; TUNEL, terminal deoxynucleotidyl transferase-mediated dUTP-biotin nick end labeling; BH3, Bcl-2 homology domain 3; GADD34, growth arrest and DNA damage gene 34; FBS, fetal bovine serum; GAPDH, glyceraldehyde-3-phosphate dehydrogenase; $\mathrm{HE}$, hematoxylin and eosin; DAPI, 4'-6-diamidino-2-phenylindole; SA- $\beta$-gal, senescence-associated $\beta$-galactosidase; X-gal, 5-bromo-4-chloro-3-indolyl- $\beta$-Dgalactopyranoside

Received 25.2.11; revised 13.6.11; accepted 16.6.11; Edited by M Blagosklonny; published online 22.7.11
} 
immunological rejection of transplants in organ transplantation. However, activation of mTORC1 is observed in a variety of other intractable pathologies including cancers, heart disease, metabolic diseases and neurodegenerative disorders. ${ }^{5,6}$ Rapamycin may be useful for the treatment of these mTORC1-related diseases. Indeed, therapeutic utility of rapamycin has been suggested for malignant diseases, diabetes, cardiovascular diseases and neurological disorders. ${ }^{7}$ Interestingly, these diseases are also considered as ER stress-related disorders. ${ }^{8-11}$ Rapamycin could exert therapeutic effects via modulating ER stress and/or subsequent UPR. However, currently, relationship between ER stress and mTORC1 remains elusive.

The serine/threonine kinase Akt/protein kinase B functions as a critical mediator for many biological functions, for example, cell proliferation, differentiation and survival. ${ }^{12}$ Akt activates mTORC1 through inactivation of tuberous sclerosis complex 2 (TSC2) within the TSC1-TSC2 complex. ${ }^{13}$ Increased activation of $\mathrm{mTORC1}$ triggers a negative feedback loop for the phosphatidylinositol 3-kinase-Akt pathway, leading to suppression of Akt. ${ }^{14,15}$ In the present investigation, we examine roles of mTORC1, Akt and the UPR in ER stress-induced apoptosis. Our current results disclose that, under ER stress conditions, activated mTORC1 causes apoptosis through suppression of Akt and consequent induction of the IRE1-JNK pathway, and that inhibition of the mTORC1 signaling attenuates ER stressinduced apoptosis in vitro and in vivo.

\section{Results}

Involvement of mTORC1 in ER stress-induced apoptosis. To investigate involvement of mTORC1 in cellular responses to ER stress, we first examined activity of mTORC1 following exposure to ER stress. Phosphorylation of p70S6K was used as an indicator for mTORC1 activation. As shown in Figure 1a, basal activity of mTORC1 was observed, and it was rapidly upregulated following the treatment with ER stress inducers thapsigargin and tunicamycin. This activation progressed time-dependently for at least $24 \mathrm{~h}$ (Figure 1b). When cells were simultaneously treated with rapamycin, the activation of mTORC1 was completely suppressed (Figures 1a and b).

We next examined an effect of rapamycin on ER stressinduced cellular death. Microscopic analysis revealed that inhibition of mTORC1 by rapamycin attenuated ER stressinduced cell death (Figures $1 \mathrm{c}$ and d). Activation of caspase-3 by tunicamycin and thapsigargin was also suppressed by the treatment with rapamycin (Figure 1e). Of note, thapsigarginand tunicamycin-induced cell death was inhibited by caspase inhibitor Z-VAD-fmk (Supplementary Figure S1). To further confirm the role of mTORC1 in ER stress-induced apoptosis, we employed RNA interference to knock down Raptor, an essential component of mTORC1. As expected, transfection with siRaptor attenuated activation of $\mathrm{mTORC} 1$ by ER stress inducers (Figure 1f). The downregulation of mTORC1 by siRaptor significantly suppressed cellular death caused by ER stress (Figures $1 \mathrm{~g}$ and $\mathrm{h}$ ). These results suggest that mTORC1 has a crucial role in the induction of apoptosis by ER stress.
Selective induction of the IRE1 pathway by mTORC1. ER stress induces apoptosis via the UPR, and the three major branches of the UPR may modulate the apoptotic process positively or negatively. ${ }^{2}$ To investigate involvement of individual UPR pathways in the pro-apoptotic effect of mTORC1, we first examined whether or not rapamycin influences ER stress-induced activation of the PERK-elF2 $\alpha$ pathway. Exposure of cells to thapsigargin or tunicamycin rapidly induced phosphorylation of PERK and elF2 $\alpha$, whereas it was unaffected by rapamycin (Figures $2 a$ and $\mathrm{b}$ ). Consistent with this result, ER stress-triggered induction of ATF4 and consequent expression of $C H O P$, a signaling event downstream of elF $2 \alpha$, was not affected by rapamycin (Figures $2 \mathrm{c}$ and $\mathrm{d}$ ). We also examined involvement of the ATF6 pathway using cleavage of p90ATF6 and consequent induction of GRP78 as indicators. ${ }^{1}$ As shown in Figures $2 e$ and f, ER stress inducers cleaved p90ATF6, and expression of GRP78 was induced following the treatment with thapsigargin and tunicamycin. However, these molecular events were not inhibited by rapamycin.

We further investigated whether activation of the IRE1 pathway is involved in the pro-apoptotic action of mTORC1. For this purpose, splicing of XBP1 mRNA and activation of the UPR element (UPRE) were used as indicators for IRE1 endoribonuclease activity. The results showed that XBP1 splicing and UPRE activation were triggered by ER stress inducers, which was significantly suppressed by the treatment with rapamycin (Figures $2 \mathrm{~g}-\mathrm{i}$ ). We also tested an effect of rapamycin on IRE1 kinase activity. The kinase domain of IRE1 activates JNK via interaction with TRAF2 and ASK1, which contributes to ER stress-induced apoptosis. ${ }^{2}$ Western blot analysis revealed that phosphorylation of JNK1 and JNK2, especially JNK1, was induced by ER stress, and it was abolished by the treatment with rapamycin (Figure 2j). Of note, the activation of JNK1 by ER stress was abrogated by dominant-negative inhibition of IRE1 (Figure 2k).

To further confirm the potential of mTORC1 to trigger IRE1 signaling, we employed RNA interference to knock down TSC2, a key negative regulator for $\operatorname{mTORC} 1 .{ }^{13}$ In the cells transiently transfected with siTSC2, TSC2 protein was downregulated, and it was correlated with activation of mTORC1 evidenced by phosphorylation of p70S6K (Figure 3a). Reporter assays showed that basal splicing of XBP1 was significantly enhanced by siTSC2 (Figure 3b). Similarly, basal phosphorylation of JNK was also reinforced by knockdown of TSC2 (Figure 3c), and it was not observed in IRE1-knockdown cells (Figure $3 d$ ). Of note, phosphorylation of PERK and elF2 $\alpha$ was not induced, and the level of GRP78 protein was unaffected by siTSC2 (Figure 3c).

The results described above were also observed under ER stress conditions. Treatment of cells with thapsigargin and tunicamycin induced splicing of XBP1 mRNA and phosphorylarion of JNK (especially JNK1), and it was enhanced by siTSC2 (Figures $3 e$ and f). In contrast, phosphorylation of PERK and elF2 $\alpha$ and induction of GRP78 were not reinforced. Under this experimental condition, enhancement of mTORC1 by siTSC2 accelerated cellular death caused by ER stress (Figures $3 \mathrm{~g}$ and $\mathrm{h}$ ). The enhancement of cell death by mTORC1 was associated with enhanced activation of 
a

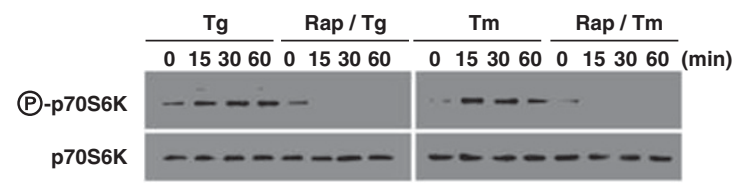

b

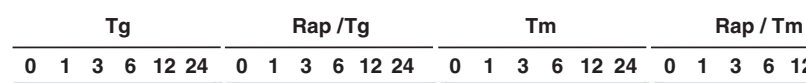

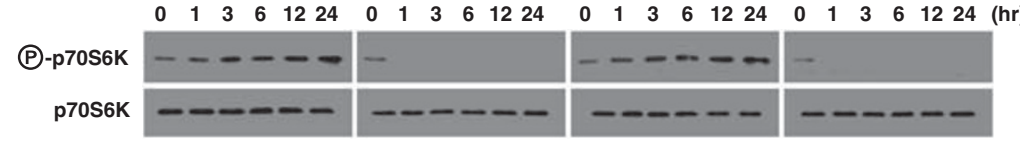

C
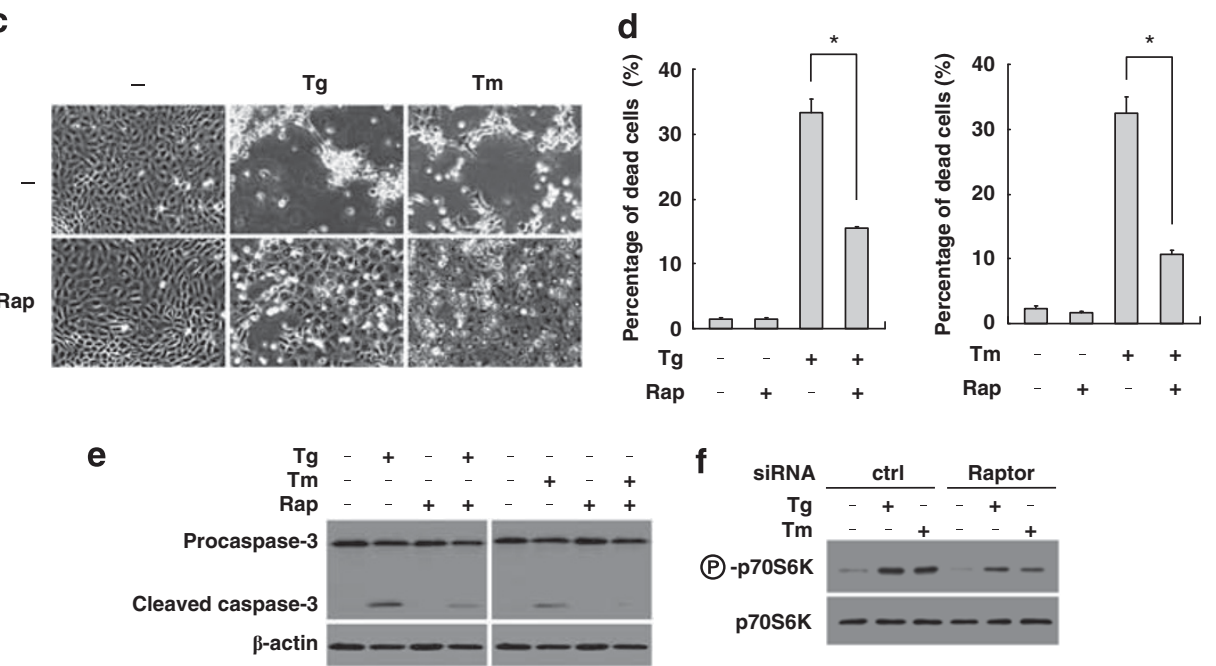

g
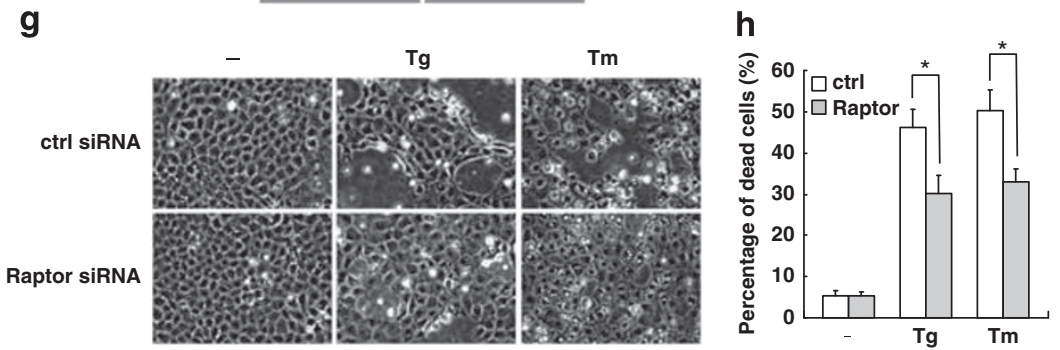

Figure 1 Involvement of mTORC1 in ER stress-induced apoptosis. (a and $\mathbf{b})$ NRK-52E cells were exposed to thapsigargin (Tg; $500 \mathrm{nM})$ or tunicamycin (Tm; $2.5 \mu \mathrm{g} / \mathrm{ml}$ ) in the absence or presence of rapamycin (Rap; $100 \mathrm{nM}$ ) for indicated time periods and subjected to western blot analysis of phosphorylated p70S6K. The level of p70S6K protein is shown at the bottom as a loading control. (c and d) Cells were treated with thapsigargin or tunicamycin in the absence or presence of rapamycin for $72 \mathrm{~h}$ and subjected to phase-contrast microscopy (c) and quantitative assessment of cell death (d). Assays were performed in quadruplicate, and data are shown as means \pm S.E. Asterisks indicate statistically significant differences $(P<0.05)$. (e) Cells were treated with indicated agents for $48 \mathrm{~h}$, and western blot analysis was performed to analyze cleavage of procaspase-3. The level of $\beta$-actin is shown at the bottom as a loading control. (f) Cells were transfected with siRaptor (20 nM) or control siRNA (ctrl; $20 \mathrm{nM}$ ), treated with indicated agents and subjected to western blot analysis of phosphorylated p70S6K. ( $(\mathbf{g}$ and $\mathbf{h}$ ) Cells were transfected with control or siRaptor, treated with ER stress inducers and subjected to phase-contrast microscopy (g) and trypan blue analysis (h)

procaspase-3 (Figure 3i). In contrast, this pro-apoptotic effect of siTSC2 was not observed in IRE1-knockdown cells (Figure 3j, Supplementary Figure S2). These data further support the idea that, under ER stress conditions, activation of mTORC1 triggers the IRE1-JNK signaling and thereby causes apoptosis.

Induction of the IRE1-JNK pathway by mTORC1 through suppression of Akt. To identify mediators for the mTORC1induced activation of IRE1, we focused on a role of Akt. There is a close link between mTORC1 and Akt; that is, Akt activates mTORC1, but activated mTORC1 may downregulate Akt signaling. ${ }^{15}$ We hypothesized that Akt might be involved in mTORC1-triggered activation of the IRE1 pathway. To examine this possibility, we first investigated whether activation of mTORC1 modulates activity of Akt. As shown in Figure 4a, activation of mTORC1 by siTSC2 caused suppression of basal Akt activity. This repression was reversed by the treatment with rapamycin (Figure $4 b$ ), confirming the potential of mTORC1 to suppress Akt.

As we previously reported, ${ }^{16}$ exposure of cells to ER stress causes transient Akt phosphorylation. Indeed, as shown in Figure 4c, treatment with thapsigargin and tunicamycin induced early phosphorylation of Akt. However, this activation was transient and biphasic. Twenty-four hours after the exposure to ER stress, the basal level of phosphorylated Akt was abolished. When the cells were treated with rapamycin, the early phosphorylation of Akt was enhanced, and late 

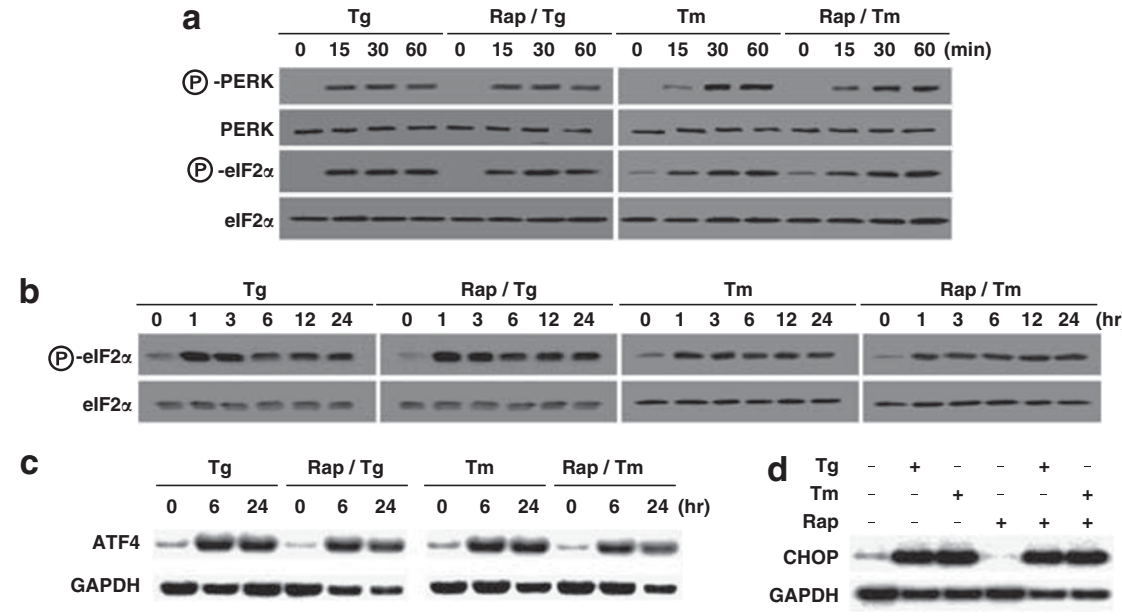

$\mathrm{Tm} \quad \operatorname{Rap} / \mathrm{Tm}$
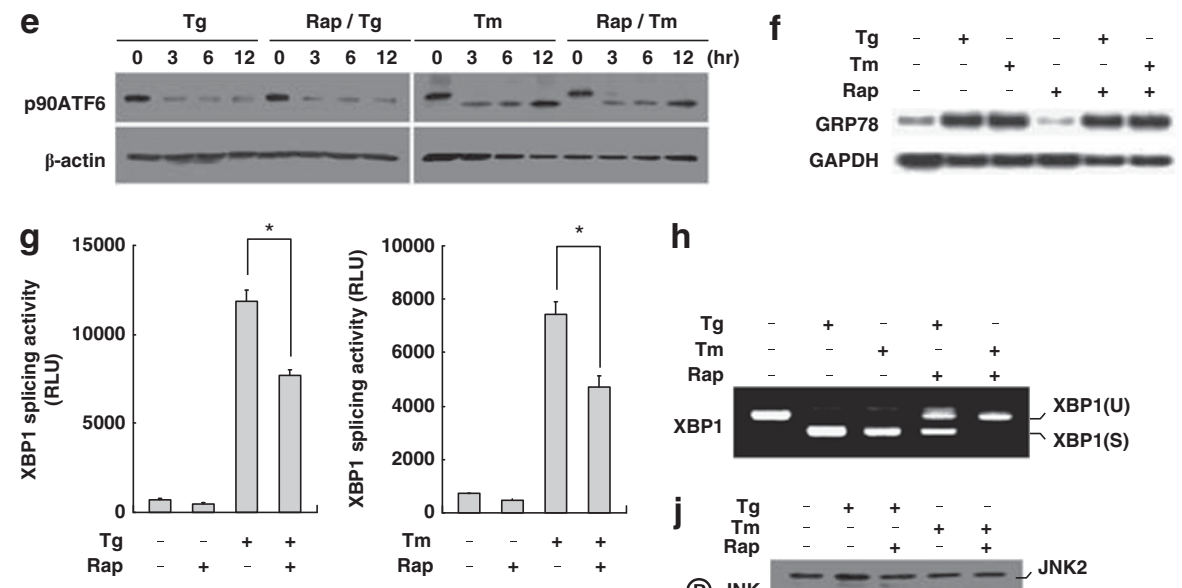

h

i

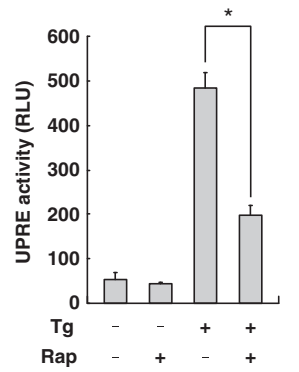

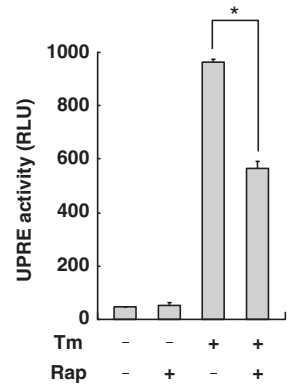
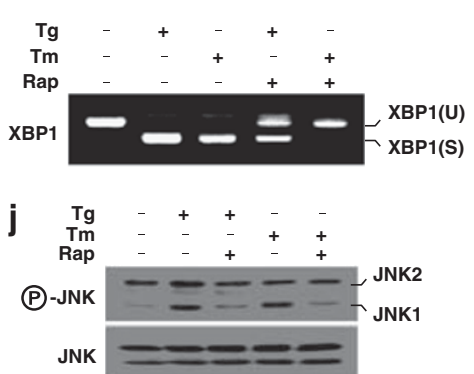

$\mathbf{k}$

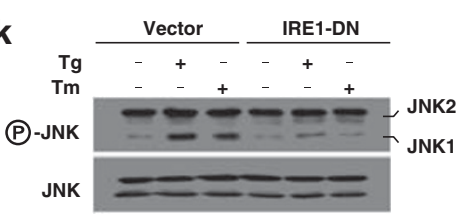

Figure 2 Selective induction of the IRE1 pathway by mTORC1. (a-f) Cells were treated with ER stress inducers together with rapamycin for indicated time periods (a-c and $\mathbf{e}$ ) or $8 \mathrm{~h}$ (d and f) and subjected to western blot analysis of phosphorylated PERK, phosphorylated elF2 $\alpha(\mathbf{a}$ and $\mathbf{b})$ and p90ATF6 (e), or northern blot analysis of ATF4 (c), CHOP (d) and GRP78 (f). As loading controls, protein levels of PERK, elF2 $\alpha$ and $\beta$-actin, and mRNA level of GAPDH were evaluated. In (e), cells were transiently transfected with FLAG-ATF6, and western blot analysis of p90ATF6 was performed using anti-FLAG antibody. ( $\mathbf{g}$ and i) Cells transiently transfected with a reporter plasmid for XBP1 splicing (g) or stably transfected NRK/UPRE-Luc cells (i) were exposed to thapsigargin or tunicamycin in the absence or presence of rapamycin for $8 \mathrm{~h}$ and subjected to chemiluminescent assay. RLU, relative light unit $\left({ }^{*} P<0.05\right)$. ( $\mathrm{h}$ and $\left.\mathrm{j}\right)$ Cells were treated with indicated agents for $4 \mathrm{~h}$ and subjected to RT-PCR analysis of $X B P 1$ splicing ( $\mathrm{h}$ ) and western blot analysis of phosphorylated JNK1 and JNK2 (j). XBP1(U), unspliced form of XBP1; XBP1(S), spliced form of XBP1. (k) NRK/Neo cells (Vector) and NRK/ IRE1-DN cells (IRE1-DN) were treated with indicated agents, and phosphorylation of JNK was evaluated. The level of JNK proteins is shown at the bottom as a loading control

dephosphorylation of Akt was inhibited, leading to its sustained activation (Figure 4c).

Under ER stress conditions, the suppression of Akt in the late phase was inversely correlated with phosphorylation of JNK1, and recovery of Akt phosphorylation by rapamycin was associated with blunted JNK activation (Figure 4d). The preserved phosphorylation of Akt by rapamycin was also observed in glomerular mesangial cells (Supplementary Figure S3). Consistent with these results, inhibition of mTORC1 by siRaptor resulted in elevation of phosphorylated Akt and blunted activation of JNK in ER stress-exposed cells (Figure 4e).

To examine a link between Akt and the IRE1 pathway, we investigated whether inhibition of Akt results in induction of IRE1 signaling. For this purpose, Akti-1/2, a selective inhibitor 

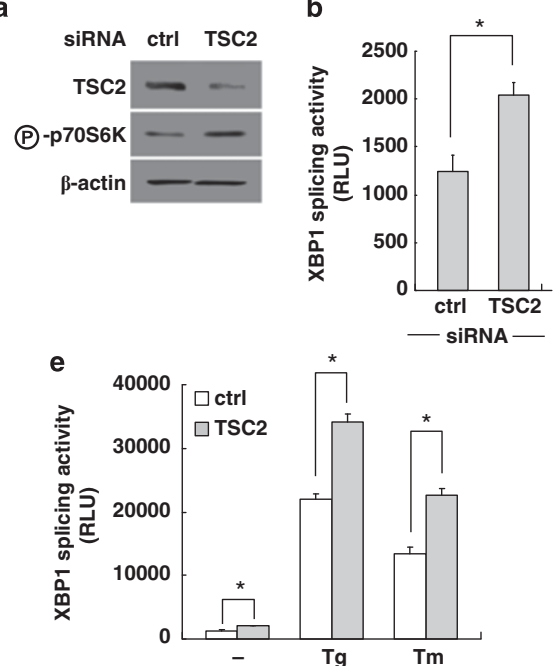

C

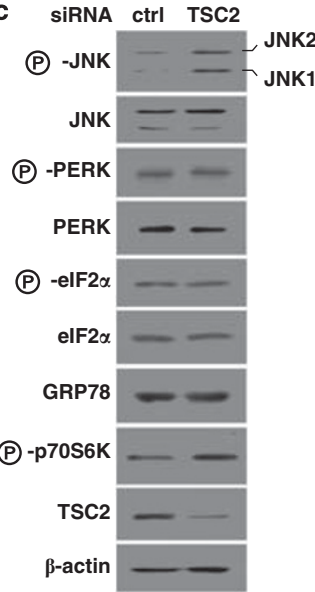

d
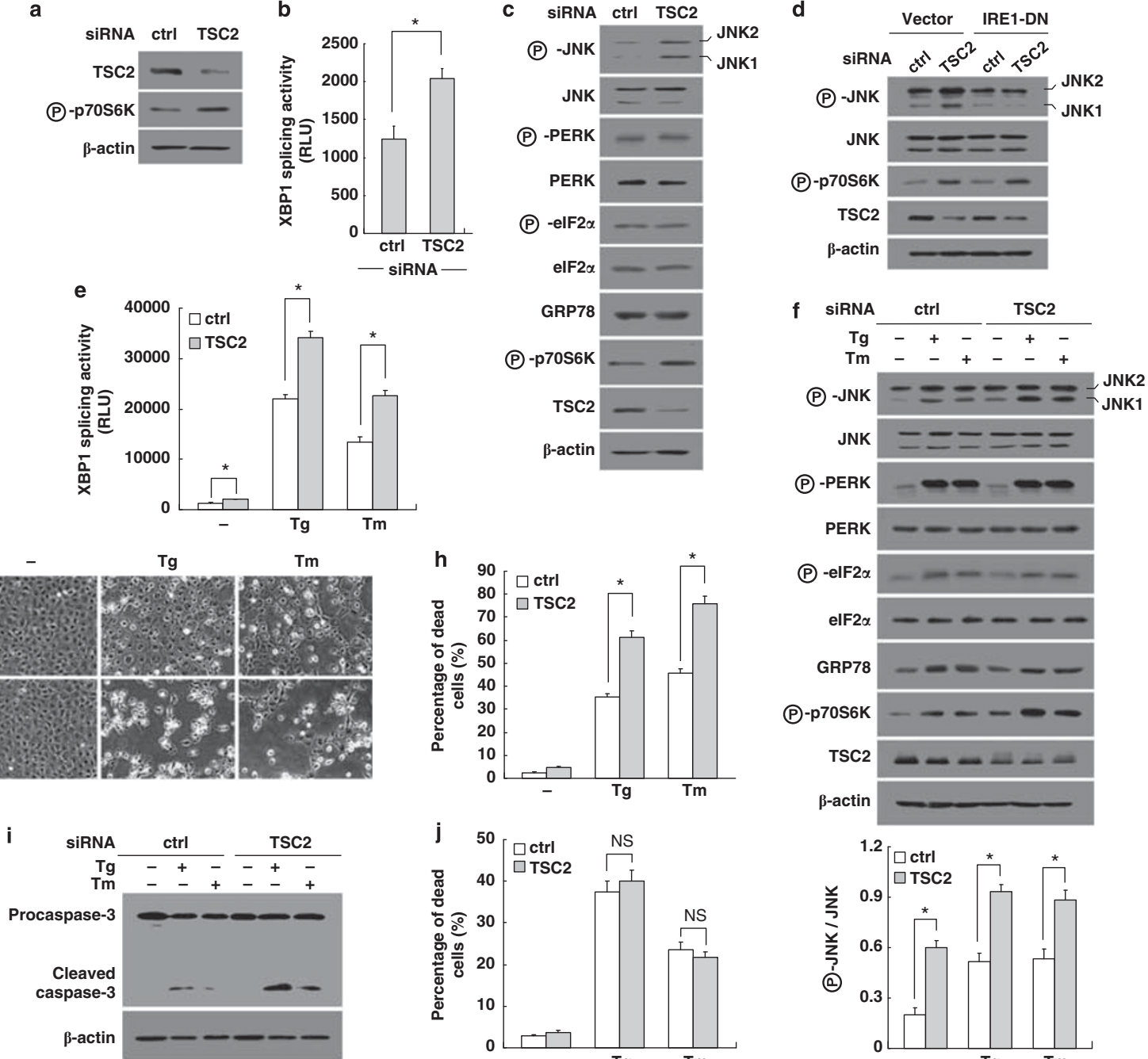
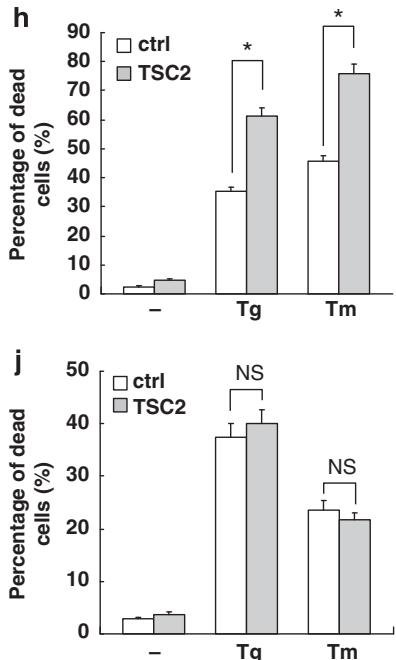

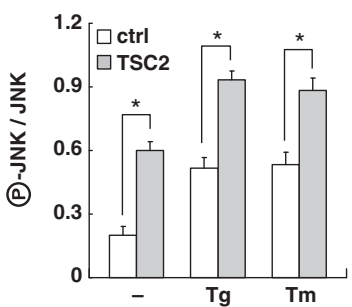

Figure 3 Induction of the IRE1-JNK pathway and enhancement of ER stress-induced apoptosis by mTORC1. (a and c) Cells were transfected with control siRNA or siTSC2 $(20 \mathrm{nM})$ for $72 \mathrm{~h}$ and subjected to western blot analysis of the molecules indicated on the left. (b and e) Cells were co-transfected with the XBP1 reporter plasmid together with indicated siRNA, treated without (b) or with (e) ER stress inducers and subjected to chemiluminescent assay ${ }^{*} P<0.05$ ). (d) NRK/Neo cells and NRK/IRE1-DN cells were transfected with indicated siRNA and subjected to western blot analysis of phosphorylated JNK and p70S6K. (f) Cells transfected with siRNA were treated with ER stress inducers and subjected to western blot analysis of the molecules indicated on the left. The level of phosphorylated JNK1/2 against total JNK proteins is shown in the bottom graph $(n=3)$. $(\mathbf{g}-\mathbf{i})$ Cells were transfected with siRNA, treated with indicated agents and subjected to phase-contrast microscopy $(\mathbf{g})$, trypan blue analysis $(\mathbf{h})$ and western blot analysis of caspase-3 (i). (j) NRK/IRE1-DN cells were transfected with siRNA, treated with ER stress inducers and subjected to trypan blue analysis. NS, not statistically significant

of Akt1 and Akt2, was used. When cells were treated with Akti$1 / 2$, basal and ER stress-induced IRE1 activity was upregulated (Figures $5 \mathrm{a}$ and $\mathrm{b}$ ). Furthermore, suppression of IRE1 activation by rapamycin was reversed by the inhibition of Akt (Figure 5c). Consistent with these results, the suppressive effect of rapamycin on phosphorylation of JNK1 and JNK2 was also reversed by the blockade of Akt activation (Figure $5 \mathrm{~d}$ ). Of note, treatment of cells with Akti-1/2 enhanced basal phosphorylation of JNK1 and JNK2. The stimulatory effect of Akti-1/2 on JNK was not observed in IRE1-knockdown cells (Figure 5e).

We tested the effects of Akt inhibition on ER stress-induced apoptosis. As shown in Figure 5f, rapamycin attenuated ER stress-induced cell injury, and this pro-survival effect was abolished by the treatment with Akti-1/2. ER stress-induced apoptosis was enhanced by inhibition of Akt (Figure $5 \mathrm{~g}$ ). The antiapoptotic effect of rapamycin was reversed by inhibition of Akt in ER stress-exposed cells (Figure 5h). Consistent with these results, suppression of caspase- 3 activation by rapamycin was abrogated in the presence of Akti-1/2 (Figure 5i). Of note, the pro-apoptotic effect of Akti-1/2 was not observed in IRE1-knockdown cells (Figure 5j, Supplementary Figure S4).

To further confirm our conclusion, another set of experiments - gain-of-function studies - were performed using overexpression of constitutively active Akt (Akt-CA). As shown in Figure 6a, ER stress-induced activation of IRE1 was attenuated by transfection with Akt-CA. Consistently, phosphorylation of JNK by ER stress inducers was also attenuated by Akt-CA (Figure $6 \mathrm{~b}$ ). The reduction in the activity of the IRE1-JNK pathway was correlated with attenuated ER stress-induced cell death (Figures $6 \mathrm{c}$ and $\mathrm{d}$ ). Furthermore, Akt-CA inhibited activation of caspase-3 by ER stress 

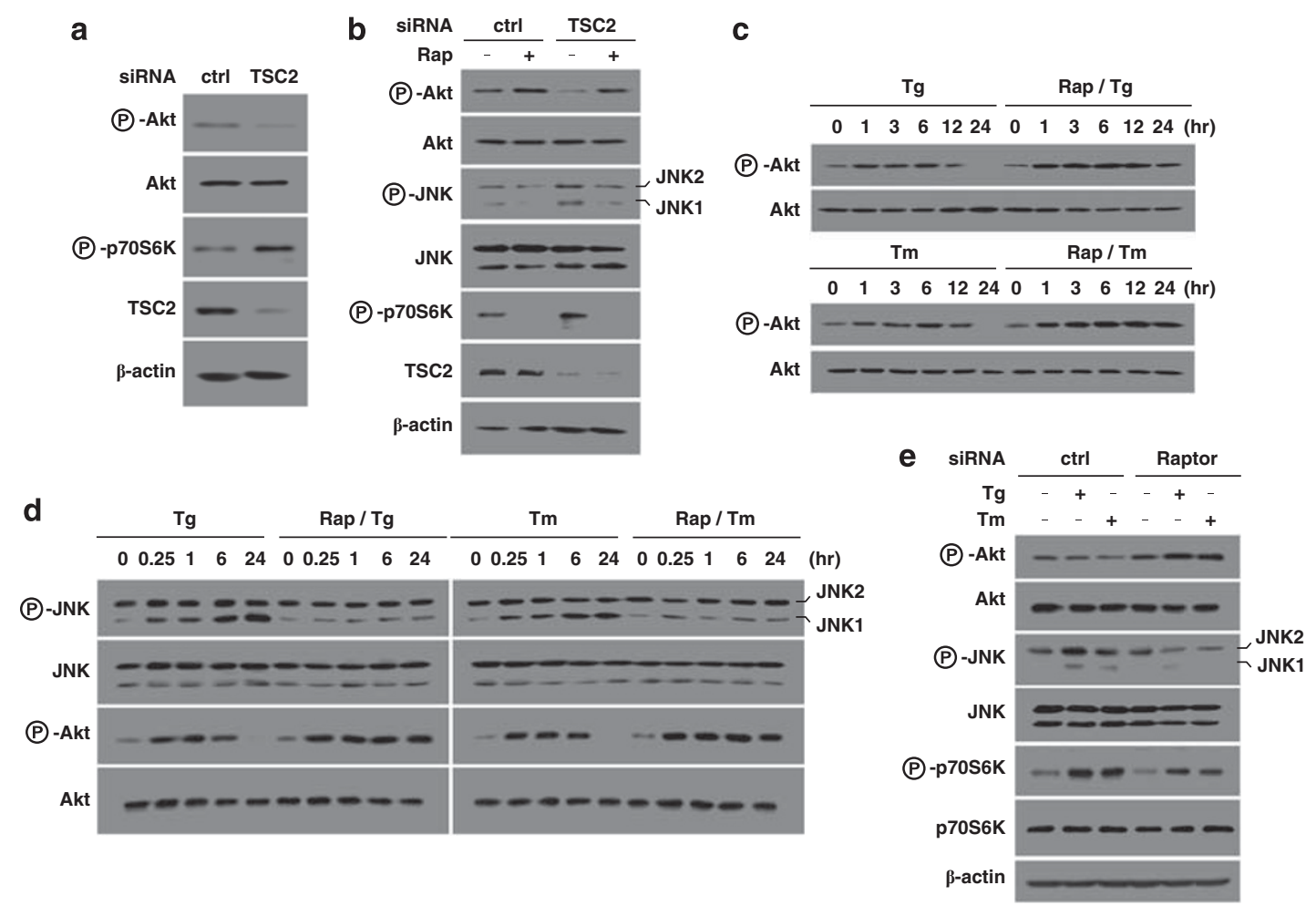

Figure 4 Suppression of Akt phosphorylation by mTORC1. (a) Cells were transfected with control siRNA or siTSC2, and phosphorylation of Akt was analyzed by western blot analysis. The protein level of Akt is shown at the bottom as a loading control. (b) Cells transfected with siRNA were treated with or without rapamycin for $24 \mathrm{~h}$, and phosphorylation of Akt and JNK was evaluated. (c) Cells were exposed to thapsigargin or tunicamycin in the absence or presence of rapamycin for the indicated time periods and subjected to western blot analysis of phosphorylated Akt. (d) Cells were exposed to thapsigargin $(100 \mathrm{nM})$ or tunicamycin $(0.5 \mu \mathrm{g} / \mathrm{ml})$ in the absence or presence of rapamycin for indicated time periods and subjected to western blot analysis. (e) Cells transfected with control siRNA or siRaptor were treated with ER stress inducers for $12 \mathrm{~h}$ and subjected to western blot analysis of indicated molecules

inducers (Figure 6e). Taken together, these results support our conclusion that $\mathrm{MTORC} 1$ induces the IRE1-JNK pathway by suppression of Akt.

IRE1 induces the ASK1-JNK pathway via interaction with TRAF2. To elucidate molecular mechanisms underlying the link between Akt and IRE1, we brought TRAF2 into focus. Western blot analysis showed that TRAF2 was constitutively expressed in unstimulated cells, and it was enhanced by the treatment with Akti-1/2 (Figure 6f). On the contrary, transfection with Akt-CA downregulated the level of TRAF2 (Figure 6g). These results suggest a possibility that Akt downregulates TRAF2 and thereby blocks the IRE1 signaling, which may be involved in the suppressive effect of rapamycin on JNK. Indeed, rapamycin markedly suppressed the level of TRAF2 under ER stress conditions (Figure 6h). Furthermore, activation of mTORC1 by siTSC2 increased the level of TRAF2. It was correlated with enhanced phosphorylation of JNK, especially JNK1 (Figure 6i), supporting our hypothesis.

In vivo suppression of ER stress-induced apoptosis by blockade of mTORC1. To evaluate in vivo effects of mTORC1 inhibition on ER stress-induced apoptosis, mice were treated with or without rapamycin and exposed to ER stress by intraperitoneal injection of tunicamycin. After three days, kidneys and livers were subjected to western blot analysis. As shown in Figure 7a, activation of mTORC1 was induced by tunicamycin in kidneys, and it was suppressed by the administration with rapamycin. Similar results were also observed in livers (Supplementary Figure S5). Consistent with our in vitro results, the suppression of mTORC1 was correlated with suppression of JNK and activation of Akt. Of note, although phosphorylation of elF2 $\alpha$ was evident in kidneys and livers in tunicamycin-exposed mice, it was not affected by the treatment with rapamycin (Figure 7a, Supplementary Figure S5).

Tunicamycin induces tubular injury and apoptosis in murine kidneys. ${ }^{17}$ Indeed, histopathological analysis evidenced vacuolation of the renal proximal tubules (Figure $7 \mathrm{~b}$, righttop), and administration of rapamycin suppressed this pathological change (Figure 7b, right-bottom). Furthermore, terminal deoxynucleotidyl transferase-mediated dUTP-biotin nick end labeling (TUNEL) staining exhibited numerous TUNEL-positive cells in kidneys of tunicamycin-exposed mice, and the number of apoptotic cells was substantially reduced in tunicamycin/rapamycin-treated mice (Figure 7c). Quantitative analysis showed that the number of TUNELpositive cells was significantly reduced by the administration with rapamycin from $193 \pm 44$ cells/field to $55 \pm 8$ cells/field (Figure $7 \mathrm{~d}$ ). These results provide in vivo evidence supporting our conclusion that, under the ER stress condition, mTORC1 causes apoptosis through suppression of Akt and consequent induction of the IRE1-JNK pathway.

\section{Discussion}

Previous reports suggested that rapamycin, the potent immunosuppressant, may be useful for the treatment of ER 

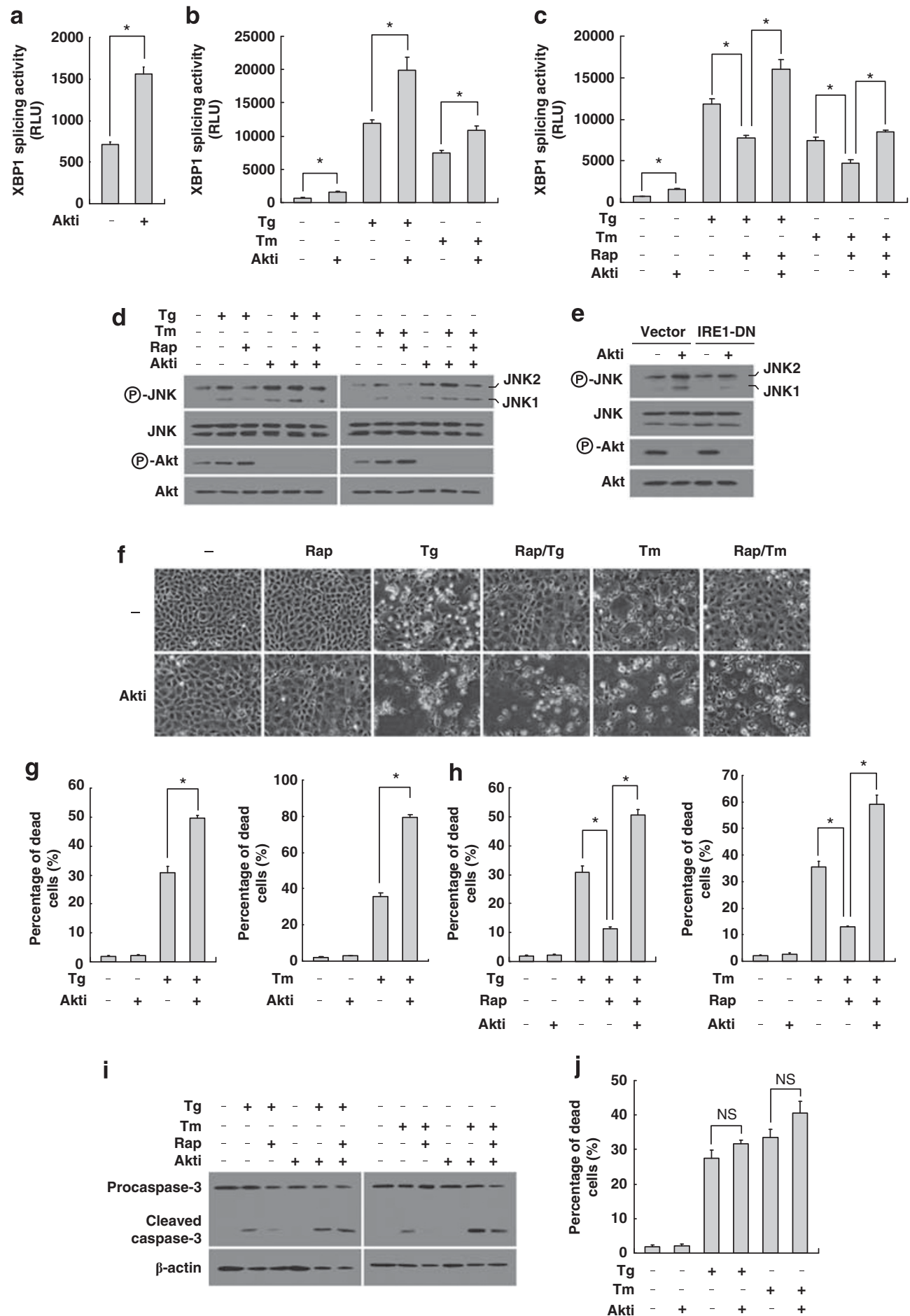

Figure 5 Activation of the IRE1-JNK pathway and enhancement of apoptosis by inhibition of Akt. (a-c) Cells were transiently transfected with the XBP1 reporter plasmid, treated with an inhibitor of Akt (Akti-1/2, indicated as Akti; $10 \mu \mathrm{M}$ ) in combination with indicated agents for $8 \mathrm{~h}$ and subjected to chemiluminescent assay $\left({ }^{\star} P<0.05\right)$. (d) Cells were treated with indicated agents and subjected to western blot analysis of phosphorylated JNK and Akt. (e) NRK/Neo cells and NRK/RE1-DN cells were treated with Akti-1/2 for $24 \mathrm{~h}$ and subjected to western blot analysis. ( $\mathbf{f}-\mathbf{i})$ Cells were treated with indicated agents and subjected to phase-contrast microscopy (f), trypan blue analysis $(\mathbf{g}$ and $\mathbf{h})$ and western blot analysis of caspase-3 (i). (j) NRK/IRE1-DN cells were treated with indicated agents and subjected to trypan blue analysis

stress-related disorders. ${ }^{7}$ However, molecular mechanisms underlying its therapeutic utility are largely unknown. In the present report, we elucidated the potential of rapamycin to attenuate ER stress-induced apoptosis. Our results dis- closed; (1) mTORC1 is activated under ER stress conditions, (2) inhibition of mTORC1 selectively suppresses the IRE1JNK pathway, but not the PERK and ATF6 pathways, and (3) activation of mTORC1 triggers the IRE1-JNK pathway, but 
a

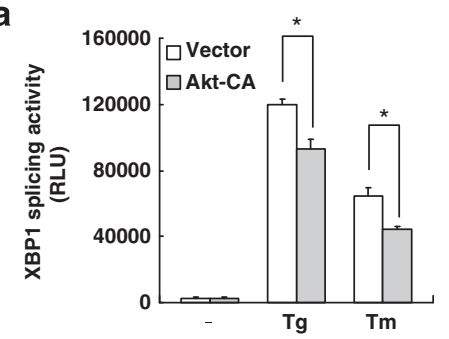

b

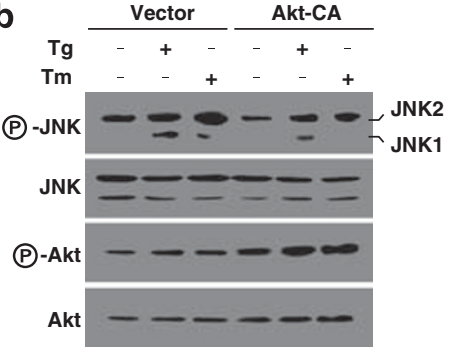

C
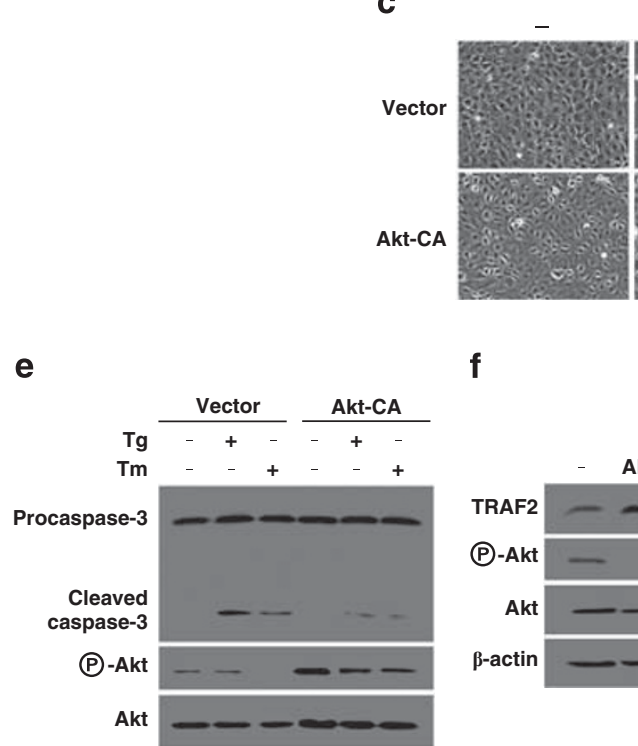

f

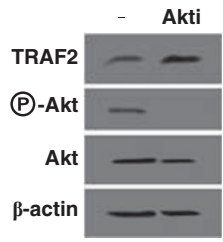

$\mathrm{Tg}$

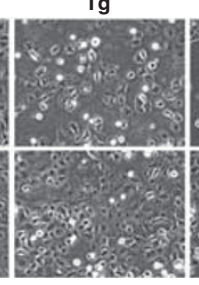

Tm

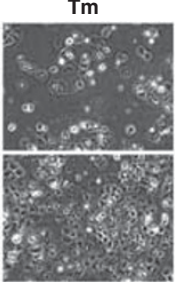

d

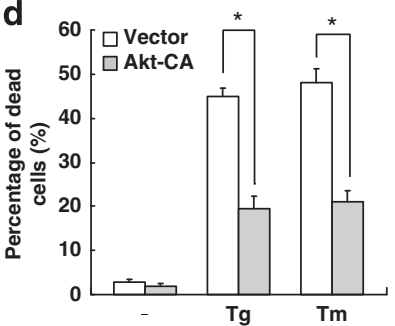

g

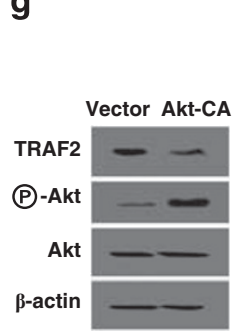

h

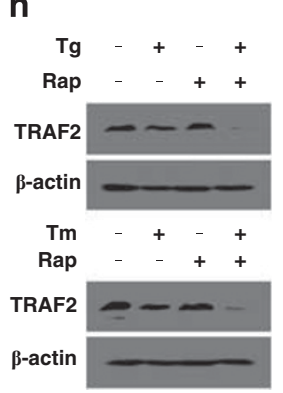

i

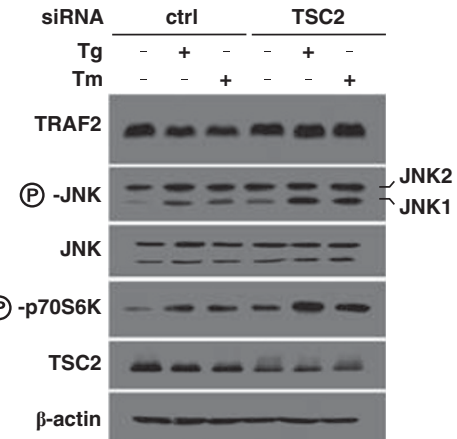

Figure 6 Suppression of the IRE1-JNK pathway and apoptosis by activation of Akt. (a) Cells were co-transfected with the XBP1 reporter plasmid together with Akt-CA, exposed to thapsigargin or tunicamycin and subjected to luciferase assay $\left({ }^{*} P<0.05\right)$. (b-e) Cells were transfected with empty vector (Vector) or $A k t-C A$, treated with ER stress inducers and subjected to western blot analysis ( $\mathbf{b}$ and $\mathbf{e}$ ), phase-contrast microscopy (c) and trypan blue assay (d). ( $\mathbf{f}$ and $\mathbf{g}$ ) Cells were treated with Akti-1/2 for $24 \mathrm{~h}$ (f) or transfected with $A k t-C A$ for $48 \mathrm{~h}(\mathbf{g})$ and subjected to western blot analysis of TRAF2 and phosphorylated Akt. (h) Cells were treated with indicated agents, and the level of TRAF2 protein was evaluated. (i) Cells transfected with control siRNA or siTSC2 were treated with ER stress inducers and subjected to western blot analysis of the molecules indicated on the left

not other UPR branches. We also elucidated that mTORC1 causes inhibition of Akt and consequent activation of the IRE1-JNK pathway, leading to apoptosis. These results suggest that, under ER stress conditions, mTORC1 causes apoptosis through suppression of Akt and consequent induction of the particular pro-apoptotic pathway. The outline of our current findings was summarized in Figure 7e.

Some previous reports showed that activation of mTORC1 resulted in induction of ER stress. For example, Ozcan et al. reported that loss of TSC1 or TSC2 and consequent activation of mTORC1 caused ER stress, leading to increased vulnerability of cells to apoptotic cell death. ${ }^{18}$ The authors showed that three major branches of the UPR were activated in TSCdeficient cells. In contrast, in the present report, we showed that activation of mTORC1 by knockdown of TSC2 triggered the IRE1-JNK pathway, but not PERK and ATF6 pathways. The discrepancy between our current results and the previous finding is possibly owing to the level of mTORC1 activation. That is, complete deletion of TSC results in strong, constitutive activation of $\mathrm{mTORC} 1$ and sustained overproduction and attenuated degradation of proteins, ${ }^{19}$ leading to activation of three branches of the UPR. In contrast, as we demonstrated, partial knockdown of TSC does not trigger substantial ER stress and causes activation of the IRE1 pathway without induction of PERK and ATF6 signaling. The fact that GRP78, the most popular ER stress marker, was not induced by the treatment with siTSC2 supports our speculation.

In the present report, we demonstrated that ER stress triggers activation of mTORC1. What is the event upstream of mTORC1 activation? One possible answer is Akt. As demonstrated in this report, ER stress rapidly induces phosphorylation of Akt. However, this early activation is only transient. In the later phase, the phosphorylation level of Akt is depressed below basal phosphorylation levels. It is caused by the negative feedback loop initiated by mTORC1, because rapamycin abolished the suppression of Akt in the late phase. It is known that mTORC1 is upregulated by $\mathrm{Akt}^{12}$ The activation of mTORC 1 by ER stress is possibly mediated by the early phosphorylation of Akt. Huang and Manning reported that mTORC2 activation was severely blunted in mTORC1activated cells. On the other hand, suppression of mTORC1 increased mTORC2 kinase activity. ${ }^{20}$ mTORC2 is known to serve as an upstream activator of Akt via direct phosphorylation at S473. ${ }^{21}$ The ability of mTORC2 to phosphorylate Akt is 
a

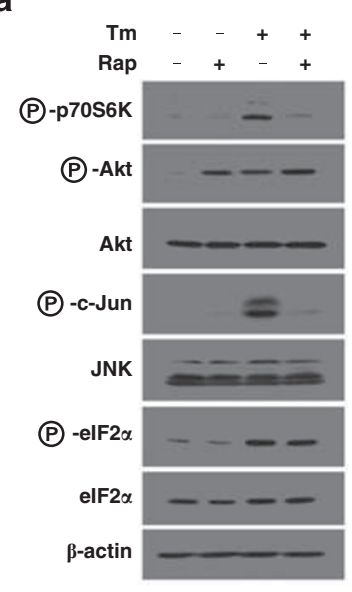

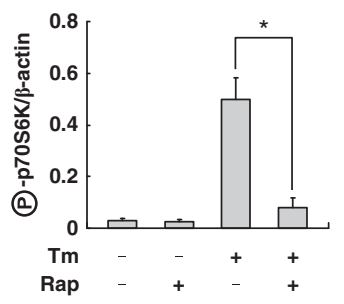

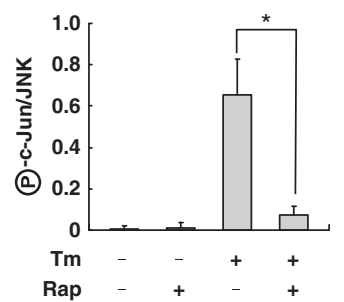

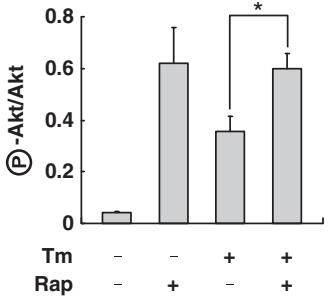

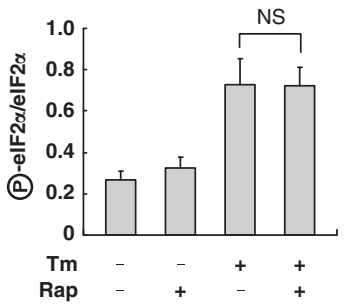

b

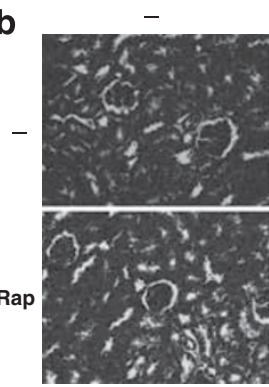

$\mathrm{Tm}$

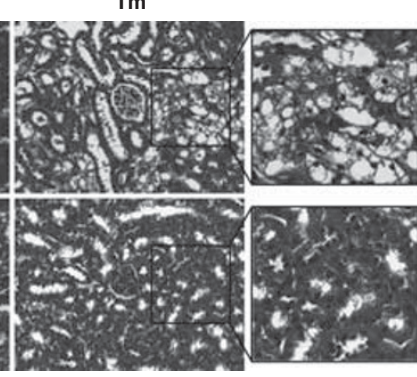

c

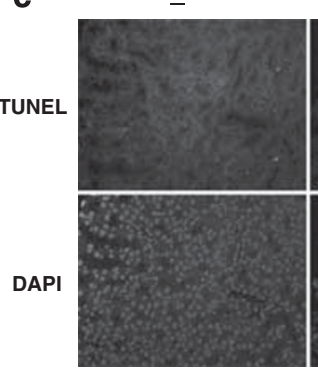

Rap

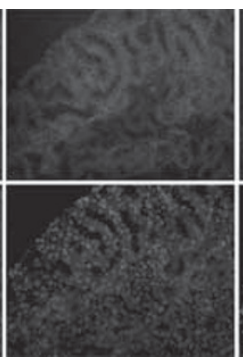

Tm

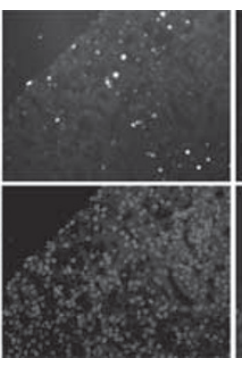

Rap/Tm
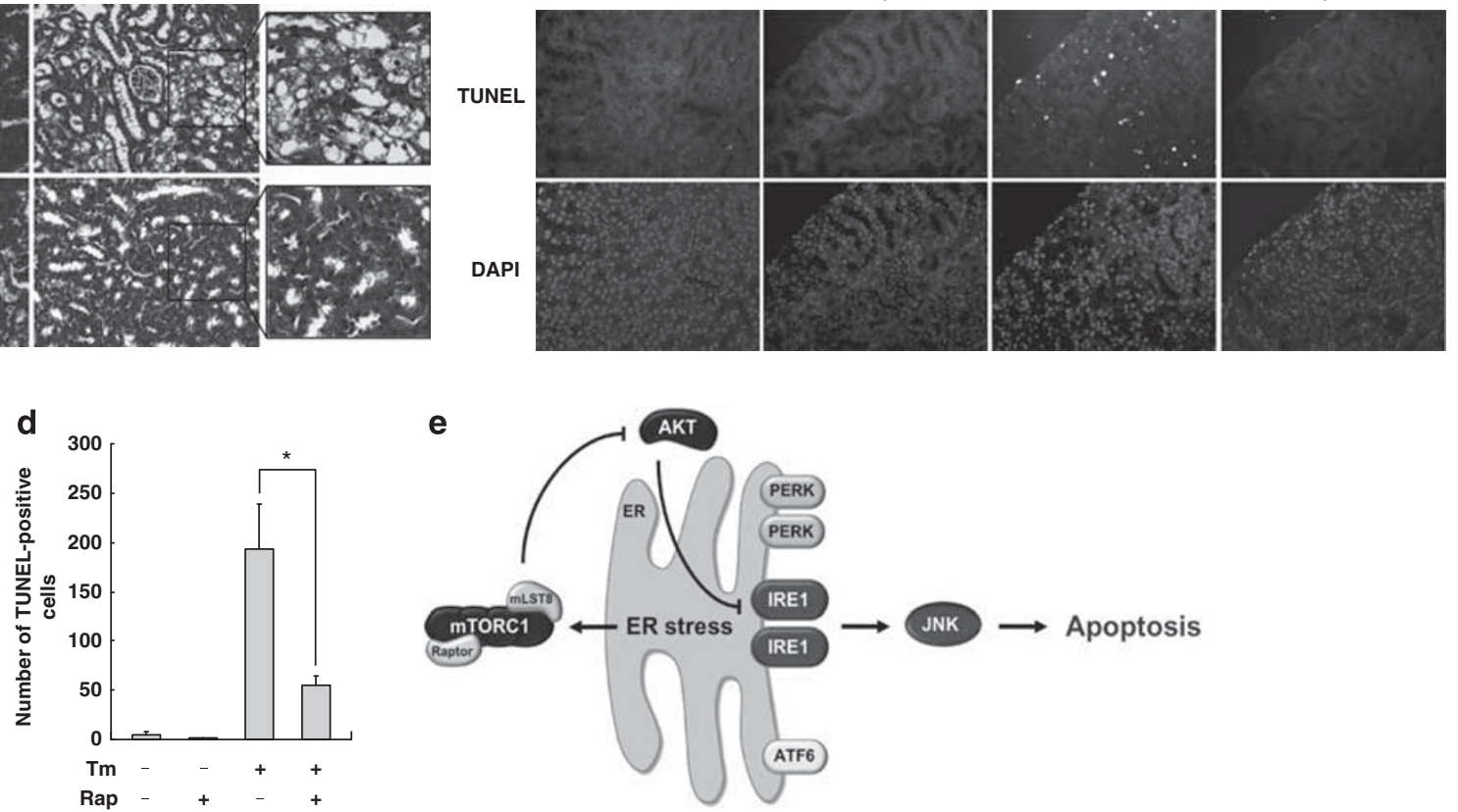

Figure 7 In vivo suppression of ER stress-induced IRE1-JNK pathway and renal tubular injury by blockade of mTORC1. (a-c) Mice were injected with PBS or rapamycin $(1.5 \mathrm{mg} / \mathrm{kg})$ intraperitoneally on day $1,2,3$ and 4 . On day 2, mice were administered with tunicamycin (1.5 mg/kg). After three days (day 5), kidneys were subjected to western blot analysis of molecules indicated on the left (a), histopathological analysis (HE staining) (b) and TUNEL assay (c). In (a), quantitative analysis of individual bands is shown in the right graphs. DAPI was used for nuclear staining shown in (c). (d) The number of TUNEL-positive cells per field was evaluated quantitatively. Data are expressed as means \pm S.E., and asterisks indicate statistically significant differences $(P<0.05)$. (e) A pro-apoptotic role of mTORC1 in ER stress-induced apoptosis. mTORC1 is activated under ER stress conditions, leading to suppression of Akt phosphorylation. As Akt suppresses activation of IRE1, mTORC1-mediated inhibition of Akt causes selective activation of the IRE1-JNK pathway and induces apoptosis. The mTORC1-Akt signaling does not affect PERK and ATF6 branches of the UPR

negatively regulated by mTORC1-mediated activation of p70S6K. ${ }^{22}$ Based on these previous findings, activation of mTORC1 by ER stress may lead to suppression of mTORC2 and consequent inhibition of Akt.

Three major branches of the UPR are activated in response to ER stress. Chemical inhibitors of ER stress (e.g., chemical chaperones) usually block all three arms of the UPR. Currently, little is known about agents that selectively inhibit particular UPR branches. In the present report, we demonstrated the potential of rapamycin for selective inhibition of the IRE1 pathway. As rapamycin did not affect ER stress-induced activation of the PERK and ATF6 pathways, and downstream expression of $C H O P$ and GRP78, its suppressive effect on the IRE1-JNK pathway is not via attenuation of ER stress per se. If so, how does Akt suppress the IRE1 pathway selectively? Currently, the mechanism is not fully understood, but our current results indicate possible involvement of TRAF2, an essential component for IRE1-mediated ASK1-JNK activation. ${ }^{23}$ We found; (1) inhibition of mTORC1 by rapamycin upregulated Akt and downregulated TRAF2, (2) activation of mTORC1 by siTSC2 downregulated Akt and upregulated TRAF2, (3) activation of Akt decreased TRAF2, whereas inhibition of Akt increased TRAF2, and (4) under ER stress conditions, the kinetics of TRAF2 levels was closely corre- 
lated with the kinetics of JNK activity. These results indicate that suppression of TRAF2 is, at least in part, responsible for Akt-mediated inhibition of JNK activation.

The action of IRE1 as kinase requires TRAF2, whereas its action as endoribonuclease does not. The fact that Akt inhibited splicing of XBP1 mRNA implies additional mechanisms underlying the suppression of IRE 1 by Akt. Previous studies suggested that Bcl-2 family proteins including Bax, Bak and Bcl-2 homology domain $3(\mathrm{BH} 3)$-only proteins directly interacted with IRE1 and regulated both its kinase and endoribonuclease activity. ${ }^{24}$ Akt is known to directly phosphorylate and inhibit Bax and BH3-only proteins. $^{12,25}$ The selective blockade of the IRE1 pathway by rapamycin might also be ascribed to the suppression of $\mathrm{Bcl}-2$ family members via Akt.

In this report, we examined a role of mTORC1 in ER stressinduced apoptosis. However, mTORC1 may also be involved in cellular senescence. ${ }^{26}$ Rapamycin could inhibit ER stressinduced cellular death via intervention in the senescence program. We examined this possibility using senescenceassociated $\beta$-galactosidase (SA- $\beta$-gal) as a marker. $^{27}$ However, in our experimental setting, neither thapsigargin nor tunicamycin caused senescence of NRK-52E cells (Supplementary Figure S6), excluding this possibility.

Individual UPR pathways possess both pro-apoptotic and antiapoptotic aspects. For example, the PERK pathway causes translational suppression that attenuates ER stress, whereas it also induces pro-apoptotic molecules including CHOP and GADD34 (growth arrest and DNA damage gene 34). The IRE1 pathway triggers apoptosis via activation of ASK1 and JNK, but this pathway also induces ER chaperones and ER stress-associated degradation factors, both of which attenuate ER stress. ${ }^{28}$ In general, however, the PERK and the ATF6 pathways are considered pro-survival, whereas the IRE1 pathway is regarded pro-apoptotic. ${ }^{29-32}$ A selective inhibitor of the IRE1 pathway should, therefore, have an advantage for the treatment of ER stress-related disorders. Indeed, in the present report, we showed that in vivo administration with rapamycin markedly suppressed ER stress-triggered activation of the IRE1-JNK pathway and consequent apoptosis in the kidney. Our current results raise a possibility that rapamycin may be useful for therapeutic intervention in a wide range of ER stress-related pathologies including infections, diabetes, ischemia, cancers and neurodegenerative disorders.

\section{Materials and Methods}

Reagents. Rapamycin, thapsigargin, tunicamycin, actinomycin D and 5-bromo-4chloro-3-indolyl- $\beta$-D-galactopyranoside (X-gal) were purchased from Sigma-Aldrich Japan (Tokyo, Japan). Akti-1/2 was obtained from Calbiochem (San Diego, CA, USA). Z-VAD-fmk was purchased from MBL International Corporation (Nagoya, Japan).

Cells and stable transfectants. The rat renal tubular epithelial cell line NRK-52E was purchased from American Type Culture Collection (Manassas, VA USA). The rat mesangial cell line SM43 was established as described previously. ${ }^{33}$ Cells were maintained in Dulbecco's modified Eagle's medium/Ham's F-12 (GibcoBRL, Gaithersburg, MD, USA) supplemented with $5 \%$ fetal bovine serum (FBS). All experiments were performed in the presence of $1 \%$ FBS. NRK/UPRE-LuC cells were established by stable transfection with pUPRE-Luc (provided by Dr. Laurie H Glimcher, Harvard Medical School) ${ }^{34}$ that introduces a luciferase gene under the control of the UPRE. NRK/IRE1-DN cells were established by transfection with pCAG-hIRE1 $\alpha$. K599A encoding a kinase-defective, dominant-negative mutant of IRE $1 \alpha$ (provided by Dr. Masayuki Miura, University of Tokyo). ${ }^{35}$ NRK-52E cells transfected with pcDNA3.1 (Invitrogen, Carlsbad, CA, USA) were used as a control.

Transient transfection. Using GeneJuice Transfection Reagent (Novagen, Madison, WI, USA), cells were transiently transfected with pCMV-3xFLAG-ATF6 (provided by Dr. Ron Prywes, Columbia University), ${ }^{36}$ pcDNA3-myrHA-Akt1 encoding Akt-CA (provided by Dr. Kenneth Walsh, Boston University School of Medicine), ${ }^{37}$ pCAX-F-XBP1 $\triangle$ DBD-Luc (provided by Dr. Takao Iwawaki, RIKEN), ${ }^{38}$ pMv-src or siRNAs. siTSC2 and siRaptor were purchased from TAKARA (Shiga, Japan). The nucleotide sequences are: siTSC2, 5'-GGCCCUCACAGACAAUGGA-3'; and siRaptor, $5^{\prime}$-GCCUGAGUCUGUGAAUGUA- $3^{\prime} .{ }^{39} 5^{\prime}$-GCUGCAAUCGAUUGAUA GC- $3^{\prime}$ was used as a control siRNA.

Western blot analysis. Western blot analysis was performed as described previously. ${ }^{40}$ Anti-caspase-3, anti-phospho-PERK (Thr980), Anti-PERK, anti-phosphoelF2 $\alpha$ (Ser51), anti-elF2 $\alpha$, anti-phospho-JNK (Thr183/Tyr185), anti-JNK, anti-phosphoc-Jun (Ser63), anti-phospho-p70S6K (Thr389), anti-p70S6K, anti-TSC2, anti-phosphoAkt (Ser473), anti-Akt and anti-TRAF2 antibodies were purchased from Cell Signaling Technology (Beverly, MA, USA). GRP78 was detected by anti-GRP78 antibody (Santa Cruz Biotechnology, Santa Cruz, CA, USA). Analysis of ATF6 was performed using anti-FLAG antibody (Cell Signaling). As a loading control, the level of $\beta$-actin was evaluated using anti- $\beta$-actin antibody (Sigma-Aldrich Japan). Densitometric analysis was performed using ImageJ Software (National Institutes of Health, Bethesda, MD, USA).

Northern blot analysis. Total RNA was extracted by the single-step method, and northern blot analysis was performed as described before. ${ }^{40} \mathrm{cDNAs}$ for GRP78 (provided by Dr. Kazunori Imaizumi, University of Miyazaki), ${ }^{11} \mathrm{CHOP}$ (provided by Dr. David Ron, New York University School of Medicine) and ATF4 (provided by Dr. David Ron) were used for preparation of radio-labeled probes. Expression of glyceraldehyde-3-phosphate dehydrogenase $(G A P D H)$ was used as a loading control.

RT-PCR. Reverse transcription was performed using Omniscript Reverse Transcriptase (Qiagen, Valencia, CA, USA). Splicing of XBP1 mRNA was examined using the following primers: $5^{\prime}$-ACACGCTTGGGGATGAATGC- $3^{\prime}$ and 5'-CCATGGGAAGATGTTCTGGG-3' (Sigma-Aldrich Japan).

Assessment of cell death. After exposure to ER stress, morphologic examination was performed by phase-contrast microscopy. The number of viable cells was estimated by trypan blue exclusion. Cleavage of procaspase-3 was used as another indicator for apoptosis.

Luciferase assay. Activity of luciferase was evaluated by Luciferase Assay System (Promega, Madison, WI, USA) according to the manufacturer's protocol.

Animal experiment. C57BL/6 mice (20-25 g body weight; 11 male mice) were intraperitoneally injected (i.p.) with PBS or rapamycin $(1.5 \mathrm{mg} / \mathrm{kg})$ on day $1,2,3$ and 4. On day 2, mice were exposed to ER stress by single i.p. injection of tunicamycin $(1.5 \mathrm{mg} / \mathrm{kg})$. After three days (day 5), kidneys were removed and processed for tissue sectioning for histopathological analysis and TUNEL assay, as described below. Renal cortex and liver were also used for western blot analysis.

Histopathological analysis and TUNEL assay. Kidneys were fixed in $4 \%$ phosphate-buffered paraformaldehyde overnight at $4{ }^{\circ} \mathrm{C}$ and embedded in paraffin. Tissue sections were stained with hematoxylin and eosin (HE). TUNEL assay was performed using Apoptosis Detection System, Fluorescein (Promega, Madison, WI, USA), as described previously. ${ }^{32}$ The number of TUNEL-positive cells per field was counted. 4'-6-Diamidino-2-phenylindole (DAPI; Sigma-Aldrich Japan) was used for nuclear staining.

SA- $\beta$-gal staining. Cells were fixed for $3 \mathrm{~min}$ in $3 \%$ formaldehyde and subjected to X-gal assay, as described previously. ${ }^{26}$

Statistical analysis. Reporter assays and assessment of cell death were performed in quadruplicate. Data were presented as means \pm S.E. Statistical analysis was performed by non-parametric Mann-Whitney U-test to compare data in different groups. $P$-value $<0.05$ was considered to indicate a statistically significant difference.

\section{Conflict of Interest}

The authors declare no conflict of interest. 
Acknowledgements. We thank Dr. Laurie H Glimcher (Harvard Medical School), Dr. Ron Prywes (Columbia University), Dr. Kenneth Walsh (Boston University School of Medicine), Dr. Takao Iwawaki (RIKEN), Dr. Kazunori Imaizumi (University of Miyazaki) and Dr. David Ron (New York University School of Medicine) for providing us with plasmids. We also appreciate technical assistance by Mrs Mikiko Yoda. This work was supported by Grant-in-Aids for Scientific Research from the Ministry of Education, Culture, Sports, Science and Technology, Japan (No.19651024) to M Kitamura.

1. Ron D, Walter $P$. Signal integration in the endoplasmic reticulum unfolded protein response. Nat Rev Mol Cell Biol 2007; 8: 519-529.

2. Kim R, Emi M, Tanabe K, Murakami S. Role of the unfolded protein response in cell death. Apoptosis 2006; 11: 5-13

3. Rutkowski DT, Kaufman RJ. A trip to the ER: coping with stress. Trends Cell Biol 2004; 14 : 20-28.

4. Laplante M, Sabatini DM. mTOR signaling at a glance. J Cell Sci 2009; 122: 3589-3594

5. Wullschleger S, Loewith R, Hall MN. TOR signaling in growth and metabolism. Cell 2006; 124: $471-484$

6. Garelick MG, Kennedy BK. TOR on the brain. Exp Gerontol 2011; 46: 155-163.

7. Tsang CK, Qi H, Liu LF, Zheng XF. Targeting mammalian target of rapamycin (mTOR) for health and diseases. Drug Discov Today 2007; 12: 112-124.

8. Wang G, Yang ZQ, Zhang K. Endoplasmic reticulum stress response in cancer: molecular mechanism and therapeutic potential. Am J Transl Res 2010; 2: 65-74.

9. Hotamisligil GS. Endoplasmic reticulum stress and the inflammatory basis of metabolic disease. Cell 2010; 140: 900-917.

10. Toth A, Nickson P, Mandl A, Bannister ML, Toth K, Erhardt P. Endoplasmic reticulum stress as a novel therapeutic target in heart diseases. Cardiovasc Hematol Disord Drug Targets 2007; 7: 205-218.

11. Katayama $T$, Imaizumi $K$, Honda $A$, Yoneda $T$, Kudo $T$, Takeda M et al. Disturbed activation of endoplasmic reticulum stress transducers by familial Alzheimer's disease-linked presenilin-1 mutations. J Biol Chem 2001; 276: 43446-43454.

12. Manning BD, Cantley LC. AKT/PKB signaling: navigating downstream. Cell 2007; 129 : 1261-1274.

13. Inoki K, Li Y, Zhu T, Wu J, Guan KL. TSC2 is phosphorylated and inhibited by Akt and suppresses mTOR signalling. Nat Cell Biol 2002; 4: 648-657.

14. Inoki K, Corradetti MN, Guan KL. Dysregulation of the TSC-mTOR pathway in human disease. Nat Genet 2005; 37: 9-24.

15. Zhang HH, Lipovsky Al, Dibble CC, Sahin M, Manning BD. S6K1 regulates GSK3 under conditions of mTOR-dependent feedback inhibition of Akt. Mol Cell 2006; 24: 185-197.

16. Yamazaki H, Hiramatsu N, Hayakawa K, Tagawa Y, Okamura M, Ogata R et al. Activation of the Akt-NF- $\kappa$ B pathway by subtilase cytotoxin through the ATF6 branch of the unfolded protein response. J Immunol 2009; 183: 1480-1487.

17. Nakagawa T, Zhu H, Morishima N, Li E, Xu J, Yankner BA et al. Caspase-12 mediates endoplasmic-reticulum-specific apoptosis and cytotoxicity by amyloid- $\beta$. Nature 2000; 403 98-103.

18. Ozcan U, Ozcan L, Yilmaz E, Düvel K, Sahin M, Manning BD et al. Loss of the tuberous sclerosis complex tumor suppressors triggers the unfolded protein response to regulate insulin signaling and apoptosis. Mol Cell 2008; 29: 541-551.

19. Dennis PB, Fumagalli S, Thomas G. Target of rapamycin (TOR): balancing the opposing forces of protein synthesis and degradation. Curr Opin Genet Dev 1999; 9: 49-54.
20. Huang J, Manning BD. A complex interplay between Akt, TSC2 and the two mTOR complexes. Biochem Soc Trans 2009; 37: 217-222.

21. Huang J, Dibble CC, Matsuzaki M, Manning BD. The TSC1-TSC2 complex is required for proper activation of mTOR complex 2. Mol Cell Biol 2008; 28: 4104-4115.

22. Dibble CC, Asara JM, Manning BD. Characterization of Rictor phosphorylation sites reveals direct regulation of mTOR complex 2 by S6K1. Mol Cell Biol 2009; 29: 5657-5670.

23. Hetz C, Glimcher LH. Fine-tuning of the unfolded protein response: assembling the IRE1 $\alpha$ interactome. Mol Cell 2009; 35: 551-561.

24. Hetz C, Bernasconi P, Fisher J, Lee AH, Bassik MC, Antonsson B et al. Proapoptotic BAX and BAK modulate the unfolded protein response by a direct interaction with IRE $1 \alpha$. Science 2006; 312: 572-576.

25. Gardai SJ, Hildeman DA, Frankel SK, Whitlock BB, Frasch SC, Borregaard N et al. Phosphorylation of Bax Ser184 by Akt regulates its activity and apoptosis in neutrophils. J Biol Chem 2004; 279: 21085-21095.

26. Dimri GP, Lee X, Basile G, Acosta M, Scott G, Roskelley $C$ et al. A biomarker that identifiessenescent human cells in culture and in aging skin in vivo. Proc Natl Acad Sci USA 1995; 92: 9363-9367.

27. Demidenko ZN, Zubova SG, Bukreeva El, Pospelov VA, Pospelova TV, Blagosklonny MV Rapamycin decelerates cellular senescence. Cell Cycle 2009; 8: 1888-1895.

28. Rutkowski DT, Kaufman RJ. That which does not kill me makes me stronger: adapting to chronic ER stress. Trends Biochem Sci 2007; 32: 469-476.

29. Boyce M, Bryant KF, Jousse C, Long K, Harding HP, Scheuner D et al. A selective inhibitor of elF2 $\alpha$ dephosphorylation protects cells from ER stress. Science 2005; 307: 935-939.

30. Wiseman RL, Balch WE. A new pharmacology - drugging stressed folding pathways. Trends Mol Med 2005; 11: 347-350.

31. Thuerauf DJ, Marcinko M, Belmont PJ, Glembotski CC. Effects of the isoform-specific characteristics of ATF6 $\alpha$ and ATF6 $\beta$ on endoplasmic reticulum stress response gene expression and cell viability. J Biol Chem 2007; 282: 22865-22878.

32. Yokouchi M, Hiramatsu N, Hayakawa K, Kasai A, Takano Y, Yao J et al. Atypical, bidirectional regulation of cadmium-induced apoptosis via distinct signaling of unfolded protein response. Cell Death Differ 2007; 14: 1467-1474.

33. Kitamura M, Taylor S, Unwin R, Burton S, Shimizu F, Fine LG. Gene transfer into the rat renal glomerulus via a mesangial cell vector: Site-specific delivery, in situ amplification, and sustained expression of an exogenous gene in vivo. J Clin Invest 1994; 94: 497-505.

34. Lee AH, Iwakoshi NN, Glimcher LH. XBP-1 regulates a subset of endoplasmic reticulum resident chaperone genes in the unfolded protein response. Mol Cell Biol 2003; 23: 7448-7459.

35. Iwawaki T, Akai R, Kohno K, Miura M. A transgenic mouse model for monitoring endoplasmic reticulum stress. Nat Med 2004; 10: 98-102.

36. Shen J, Prywes R. ER stress signaling by regulated proteolysis of ATF6. Methods 2005 35: 382-389.

37. Fujio Y, Guo K, Mano T, Mitsuuchi Y, Testa JR, Walsh K. Cell cycle withdrawal promotes myogenic induction of Akt, a positive modulator of myocyte survival. Mol Cell Biol 1999; 19 : 5073-5082.

38. Iwawaki T, Akai R. Analysis of the XBP1 splicing mechanism using endoplasmic reticulum stress-indicators. Biochem Biophys Res Commun 2006; 350: 709-715.

39. Piao X, Kobayashi T, Wang L, Shiono M, Takagi Y, Sun G et al. Regulation of folliculin (the BHD gene product) phosphorylation by Tsc2-mTOR pathway. Biochem Biophys Res Commun 2009; 389: 16-21.

40. Hayakawa K, Meng Y, Hiramatsu N, Kasai A, Yamauchi K, Yao J et al. Priming of glomerular mesangial cells by activated macrophages causes blunted responses to proinflammatory stimuli. J Immunol 2006; 176: 2529-2537.

\section{Supplementary Information accompanies the paper on Cell Death and Differentiation website (http://www.nature.com/cdd)}

